\title{
Levamisole Hydrochloride
}

National Cancer Institute

\section{Source}

National Cancer Institute. Levamisole Hydrochloride. NCI Thesaurus. Code C610.

The hydrochloride salt of the synthetic imidazothiazole derivative levamisole with anthelminthic and immunomodulating activities. In immunosuppressed states, levamisole may restore immune function by: 1) stimulating antibody formation, 2) stimulating T-cell activation and proliferation, 3) potentiating monocyte and macrophage phagocytosis and chemotaxis and 4) increasing neutrophil mobility, adherence, and chemotaxis. 